\title{
Resveratrol induces apoptosis through modulation of the Akt/FoxO3a/Bim pathway in HepG2 cells
}

\author{
MI-HUA LIU ${ }^{1 *}$, XIAO-LONG LIN ${ }^{2 *}$, JIAN LI ${ }^{3 *}$, JUN HE$^{1}$, TIAN-PING TAN ${ }^{1}$, SHAO-JIAN WU ${ }^{1}$, SHAN YU ${ }^{1}$,

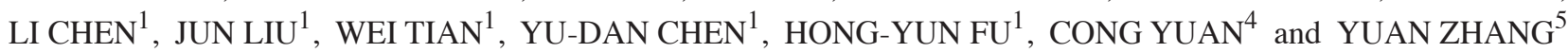 \\ ${ }^{1}$ Department of Clinical Laboratory, Affiliated Nanhua Hospital, University of South China, Hengyang, Hunan 421001; \\ ${ }^{2}$ Department of Pathology, The Third People's Hospital of Huizhou Affiliated to Guangzhou Medical University, \\ Huizhou, Guangdong 516002; ${ }^{3}$ Department of Ultrasonic Diagnosis, BoAi Hospital of Zhongshan, Zhongshan, \\ Guangdong 528403; ${ }^{4}$ Department of Cardiology, The First Hospital of Changsha, Changsha, Hunan 410005; \\ ${ }^{5}$ Department of Pathology, Mawangdui Hospital, Changsha, Hunan 410016, P.R. China
}

Received January 13, 2015; Accepted November 10, 2015

DOI: $10.3892 / \mathrm{mmr} .2015 .4695$

\begin{abstract}
Resveratrol is a polyphenolic compound found in wine, which is mainly produced by the grapevine and exerts chemopreventive effects against hepatocellular carcinoma. However, the underlying molecular mechanisms have remained to be fully elucidated. The present study assessed whether resveratrol-induced apoptosis was mediated via the activation of the forkhead box O3a (FoxO3a) transcription factor. It was demonstrated that resveratrol treatment induced apoptosis in HepG2 cells, and that this pro-apoptotic effect was accompanied with increases in the expression of apoptotic protein Bim. Following resveratrol treatment, Akt-mediated phosphorylation of FoxO3a was observed to be diminished in HepG2 cells. Furthermore, resveratrol enhanced the nuclear levels of FoxO3a and mediated neuronal death via Bim. The present study demonstrated that resveratrol induced apoptosis in HepG2 cells through activation of the transcription factor FoxO3a and increasing the expression of Bim protein.
\end{abstract}

\section{Introduction}

Hepatocellular carcinoma is one of the most common types of hepatobiliar malignancy, and features high morbidity and mortality (1). The incidence of late-stage hepatocellular

Correspondence to: Mr. Mi-Hua Liu, Department of Clinical Laboratory, Affiliated Nanhua Hospital, University of South China, 336 Dongfeng South Road, Hengyang, Hunan 421001, P.R. China E-mail:mihualiu@163.com

Miss Yuan Zhang, Department of Pathology, Mawangdui Hospital, 89 Guhan Road, Changsha, Hunan 410016, P.R. China

E-mail: 604747341@qq.com

*Contributed equally

Key words: resveratrol, high glucose, oxidative stress, apoptosis, forkhead box O3a carcinoma has been reported to increase by $>1$ million per year. Although chemotherapy and radiotherapy are usually used to treat hepatocellular carcinoma, these methods have limitations and are insufficient to abrogate its deleterious effects (2). Therefore, novel treatment strategies for hepatocellular carcinoma are urgently required.

Resveratrol is a polyphenol which occurs naturally in grapes, mulberries and peanuts (3). Resveratrol has several biological activities, including anti-inflammatory, anti-oxidant and vasorelaxant activities $(4,5)$. Resveratrol is often used as a cancer chemotherapeutic agent (6) and has further biological activities, including mimic effects of calorie restriction and delay of aging (7). Delmas et al (8) reported that resveratrol inhibited cell proliferation at micromolar concentrations in the HepG2 human hepatoblastoma cell line and the Fao rat hepatoma cell line. In addition, Parekh et al (9) reported that resveratrol downregulates cyclin D1 through inhibiting the activities of p38 mitogen-activated protein kinase and Akt, and increasing extracellular signal-regulated kinase activity to promote cell apoptosis.

A large number of studies have shown that Akt can promote cell proliferation and inhibit apoptosis, and has an important role in the occurrence and progression of tumors $(10,11)$. Forkhead box O3a transcription factor (FoxO3a) is a key downstream target of the phosphoinositide-3 kinase (PI3K)/Akt pathway. FoxO3a has been implicated in the regulation of diverse cellular functions, including proliferation, apoptosis, protection against oxidative stress and metabolism (12). Akt can promote the phosphorylation of FoxO3a, which subsequently translocates from the cell nucleus to the cytoplasm, thus inhibiting apoptosis. By contrast, inhibition of the PI3K/Akt pathway was shown to reduce the phosphorylation of FoxO3a and to thereby promote the nuclear translocation of FoxO3a to cause cell cycle arrest and apoptosis (13). Polter et al (14) showed that FoxO3a knockout mice presented with impaired development of hematopoetic stem cells. Of note, low levels of FoxO3a have been shown to be associated with resistance to chemotherapy in human cancers (15). In addition, purified vitexin compound 1 (16), melatonin (17) and casticin (18) were shown to induce apoptosis in hepatocellular carcinoma via activation of FoxO3a. 
Parekh et al (9) reported that resveratrol suppresses the growth of human HepG2 liver cancer cells by causing apoptosis; however, the mechanism of this pro-apoptotic response to resveratrol has remained elusive. Based on this information, the present study assessed the roles of the Akt/FoxO3a/Bim pathway in resveratrol-induced HepG2 liver cancer-cell apoptosis.

\section{Materials and methods}

Materials. 3-(4,5-Dime thylthiazol-2-yl)-2,5diphenyltetrazolium bromide (MTT), fibroblast growth factor 2 (FGF-2), Hoechst 33258 and resveratrol were purchased from Sigma-Aldrich (St Louis, MO, USA). The enhanced chemiluminescence (ECL) solution, paraformaldehyde, goat serum, Triton X, Tris-buffered saline with $0.1 \%$ Tween 20 (TBS-T), sodium dodecyl sulfate (SDS)-polyacrylamide gel, non-fat milk and the polyvinylidene difluoride membrane (PVDF) membrane were purchased from Beyotime Institute of Biotechnology (Haimen, China). PI3K inhibitor LY294002 was purchased from Merck Millipore (Billerica, MA, USA). All cell components of the culture medium were purchased from Thermo Fisher Scientific (Waltham, MA, USA) unless stated otherwise.

Cell culture. The HepG2 human hepatoma cell line was supplied by Sun Yat-sen University Experimental Animal Center (Guangzhou, China) and were cultured in a humidified atmosphere containing $5 \% \mathrm{CO}_{2}$ at $37^{\circ} \mathrm{C}$. HepG2 cells were seeded on six-well plates at a density of $2 \times 10^{6}$ cells/well in RPMI-1640 medium with $10 \%$ fetal bovine serum, $100 \mu \mathrm{g} / \mathrm{ml}$ streptomycin (Gibco; Thermo Fisher Scientific) and $100 \mathrm{U} / \mathrm{ml}$ penicillin-streptomycin (Gibco). HepG2 cells were passaged every two days.

MTT assay. The MTT assay was used to assess cell viability. Prior to each experiment, HepG2 cells (5,000 cells/well) were seeded in 96-well microtiter plates. HepG2 cells were treated with various concentrations of resveratrol $(0,25,50$, 100 or $200 \mu \mathrm{M})$ for $48 \mathrm{~h}$. Subsequently, $10 \mu \mathrm{l}$ MTT solution $(5 \mathrm{mg} / \mathrm{ml})$ was added to each well, followed by further incubation for $4 \mathrm{~h}$ at $37^{\circ} \mathrm{C}$. Following addition of dimethyl sulfoxide (150 $\mu$ l, Beyotime Institute of Biotechnology), the absorbance was measured at $470 \mathrm{~nm}$ with a SpectraMax 190 spectrophotometer (Molecular Devices LLC, Sunnyvale, CA, USA) and used to calculate the cell viability relative to that of the control group.

Hoechst 33258 nuclear staining for assessment of apoptosis. Hoechst 33258 was used to assess cell apoptosis. H9c2 cardiac myocytes were seeded at a density of $2 \times 10^{6}$ cells/well and incubated for $24 \mathrm{~h}$. Following treatment with resveratrol $(0,25,50$ or $100 \mu \mathrm{M}$ for $48 \mathrm{~h})$, HepG2 cells were fixed in ice-cold $4 \%$ paraformaldehyde dissolved in phosphate-buffered saline at $37^{\circ} \mathrm{C}$ for $15 \mathrm{~min} .5 \%$ normal goat serum in $0.01 \mathrm{M}$ phosphate-buffered saline (PBS) containing $0.3 \%$ Triton X-100 was used to block non-specific binding. The slides were washed three times with PBS and then incubated with $10 \mu \mathrm{g} / \mathrm{ml}$ Hoechst 33258 at $37^{\circ} \mathrm{C}$ for $15 \mathrm{~min}$. The slides were visualized under a fluorescence microscope (BX50-FLA; Olympus, Tokyo, Japan). Apoptotic cells showed condensed, fractured or distorted nuclei, while
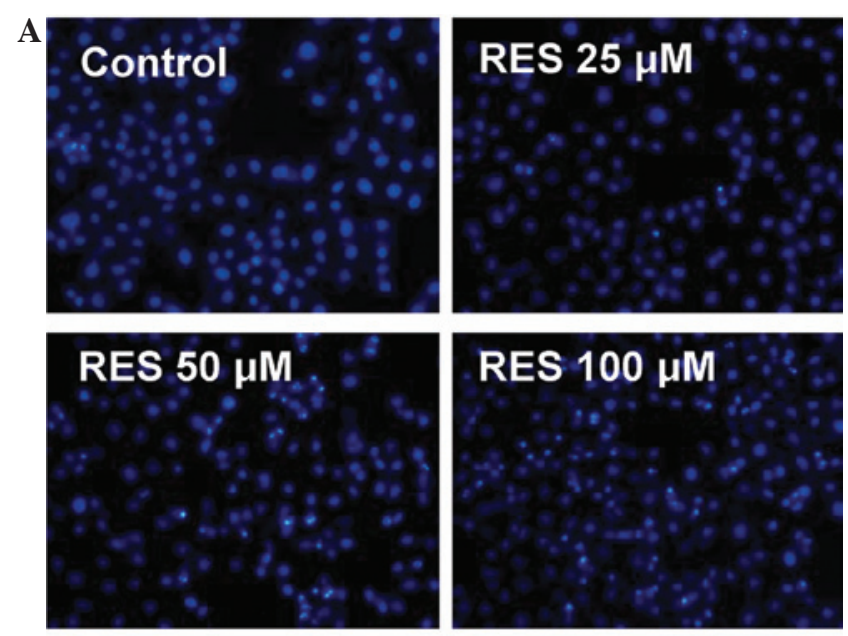

B

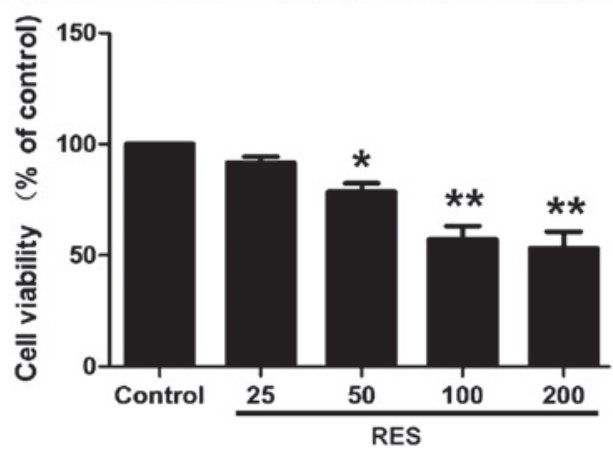

Figure 1. Effects of resveratrol treatment (48 h) on the viability of HepG2 cells. (A) Cells were stained with Hoechst 33258 (magnification, x40). (B) Quantification of viable cells in A. Values are expressed as the mean \pm standard error of the mean $(n=3)$. ${ }^{*} \mathrm{P}<0.05,{ }^{* *} \mathrm{P}<0.01$, compared with the control group. Res, resveratrol.
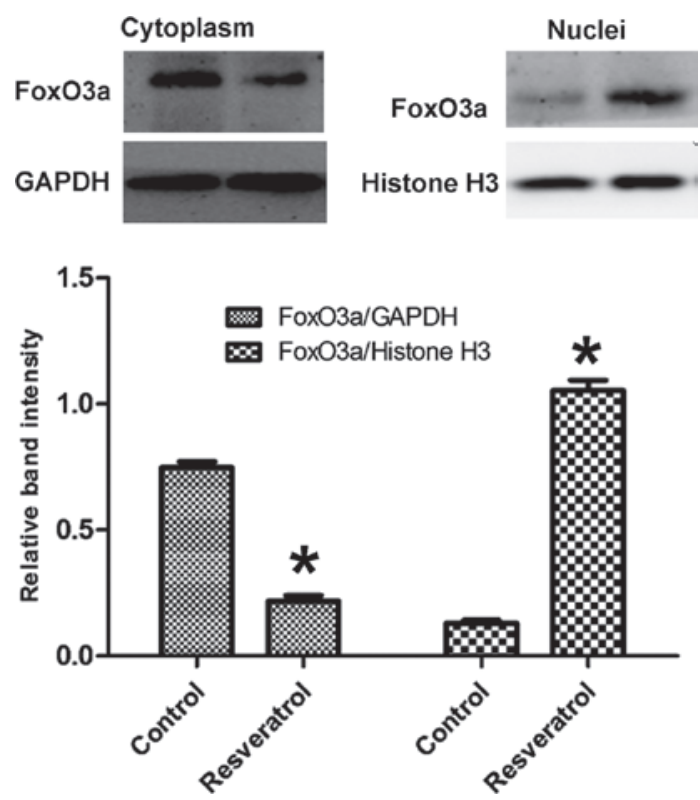

Figure 2. Effects of resveratrol treatment $(100 \mu \mathrm{M}$ for $48 \mathrm{~h})$ on cytoplasmic and nuclear protein expression of FoxO3a. Values are expressed as the mean \pm standard error of the mean $(n=3)$. ${ }^{*} \mathrm{P}<0.05$, compared with the control group. FoxO3a, forkhead box O3a.

viable cells displayed a normal nuclear size and uniform fluorescence. 
A

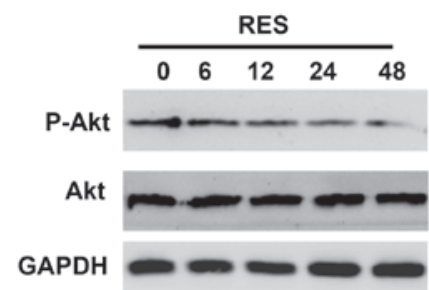

C

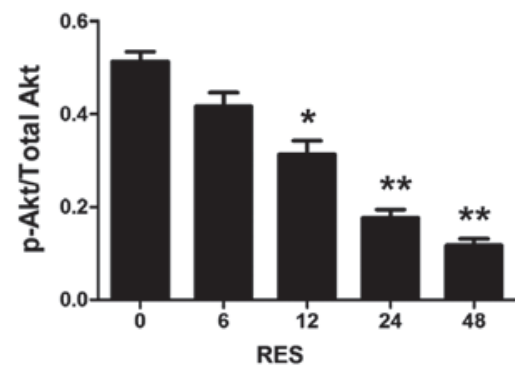

B

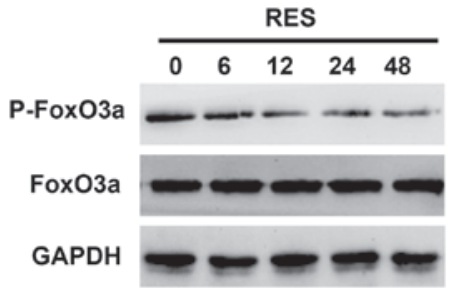

D

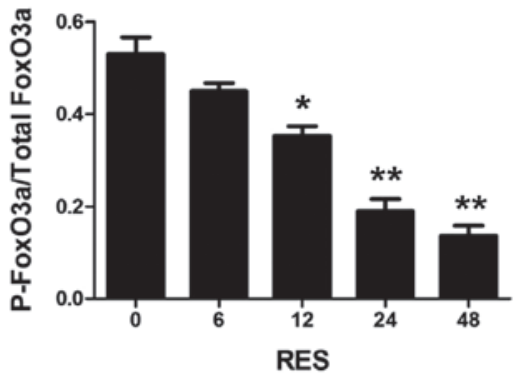

Figure 3. Effect of resveratrol on the levels of total and phosphorylated Akt and FoxO3a in HepG2 cells. (A and B) Phosphorylation of Akt and FoxO3a following treatment of HepG2 cells with resveratrol $(100 \mu \mathrm{M})$ for $0,6,12,24$ and $48 \mathrm{~h}$ were examined by western blot analysis. (C and D) Relative levels of p-FoxO3a vs. total FoxO3a and p-Akt vs. total Akt in each sample as determined by densitometry. Values are expressed as the mean \pm standard error of the mean $(n=3)$. ${ }^{*} \mathrm{P}<0.05$, compared with the control group. RES, resveratrol; p-FoxO3a, phosphorylated forkhead box O3a.

Sub-cellular fractionation and western blot analysis. HepG2 cells were incubated in $0.5 \%$ FBS DMEM for $24 \mathrm{~h}$ (control group) or treated with resveratrol $(100 \mu \mathrm{M})$ for $48 \mathrm{~h}$ (RES group), with $10 \mathrm{ng}$ FGF-2 or $10 \mathrm{~mol} / 1 \mathrm{LY} 294002$ for $30 \mathrm{~min}$ prior to exposure to resveratrol $(100 \mu \mathrm{M})$ for $48 \mathrm{~h}$ (FGF-2 + RES group or LY + RES group), or with $10 \mathrm{ng}$ FGF-2 or $10 \mu \mathrm{mol} / 1 \mathrm{LY} 294002$ for $30 \mathrm{~min}$ followed by culture for $24 \mathrm{~h}$ (FGF-2 group or LY group). Cultured HepG2 cells were fractionated into nuclear and cytoplasmic lysates using a PARIS kit (Ambion; Thermo Fisher Scientific). HepG2 cells were directly homogenized in cell lysis buffer (Cell Signaling Technology, Inc., Danvers, MA, USA) with phosphatase inhibitor cocktail (Sigma-Aldrich), and lysates were centrifuged at $14,000 \mathrm{x}$ g for $10 \mathrm{~min}$ at $4^{\circ} \mathrm{C}$. The protein concentration was determined using a bicinchoninic acid protein assay kit (Beyotime Institute of Biotechnology). The extracted proteins (30 $\mu \mathrm{g}$ per lane) were separated in $10 \%$ SDS-polyacrylamide electrophoresis gels and transferred onto a PVDF membrane. Non-specific protein binding was blocked with 5\% non-fat dried milk in TBS-T for $2 \mathrm{~h}$ at room temperature with agitation. The membranes were then incubated overnight at $4^{\circ} \mathrm{C}$ with the following diluted primary antibodies: Rabbit anti-Akt polyclonal antibody (cat. no. 9272; 1:1,000 dilution), rabbit anti-phosphorylated (p)-Akt (Ser 473) monoclonal antibody (cat. no. 4060; 1:2,000 dilution), rabbit anti-FoxO3a polyclonal antibody (cat. no. 12829; 1:1,000 dilution), rabbit anti-p-FoxO3a (Ser 253) polyclonal antibody (cat. no. 9466; 1:800 dilution) (all from Cell Signaling Technology) and rabbit anti-Bim polyclonal antibody (cat. no. ab32158; Abcam, Cambridge, MA, USA; 1:800 dilution). The membranes were then washed three times with TBS-T and incubated with horseradish peroxidase-labeled goat anti-rabbit immunoglobulin G (cat. no. A0208; Beyotime Institute of Biotechnology; 1:1,000 dilution) for $2 \mathrm{~h}$ at $37^{\circ} \mathrm{C}$. Protein bands were analyzed using visualized using an enhanced chemiluminescence reagent kit (Beyotime Institute of Biotechnology) and a western blotting detection system (Tanon-5500; Tanon Science \& Technology, Shanghai, China) and the Quantity One Software 4.6.2 Package (Bio-Rad Laboratories, Inc., Hercules, CA, USA) was used for analysis.

Statistical analysis. Values are expressed as the mean \pm standard error of the mean. Statistical analysis was performed using one-way analysis of variance with SPSS 13.0 (SPSS, Inc., Chicago, IL, USA). $\mathrm{P}<0.05$ was considered to indicate a statistically significant difference.

\section{Results}

Resveratrol reduces viability of HepG2 cells. HepG2 cells were treated with increasing doses of resveratrol for $48 \mathrm{~h}$ and subjected to an MTT assay. The results showed that resveratrol at 50-200 $\mu \mathrm{M}$ decreased the cell viability in a concentration-dependent manner (Fig. 1A). Hoechst 33258 staining further confirmed the apoptosis-inducing potential of resveratrol (Fig. 1B). Resveratrol treatment resulted in a significant increase in the apoptotic rate of the cardiomyocytes. As $100 \mu \mathrm{M}$ resveratrol induced a significant reduction in cell viability and promoted apoptosis in HepG2 cells, this concentration was selected for use in the subsequent experiments.

Resveratrol treatment causes nuclear translocation of FoxO3a. To investigate the role of resveratrol on the sub-cellular location of FoxO3a, nuclear and cytosolic protein fractions of HepG2 cells were obtained and subjected to western blot analysis of FoxO3a (Fig. 2). Following treatment with resveratrol, the protein levels of FoxO3a were increased in the nucleus, but decreased in the cytoplasm, indicating that resveratrol caused nuclear translocation of FoxO3a.

Resveratrol decreases the phosphorylation of Akt and FoxO3a in HepG2 cells. To investigate the roles of the 
A

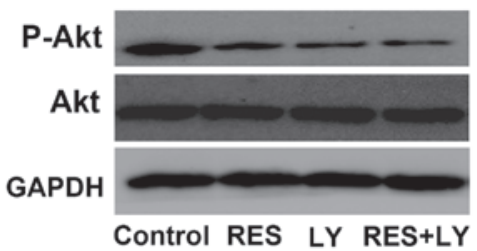

C

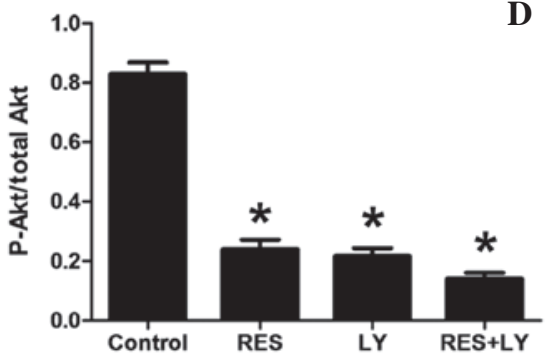

B

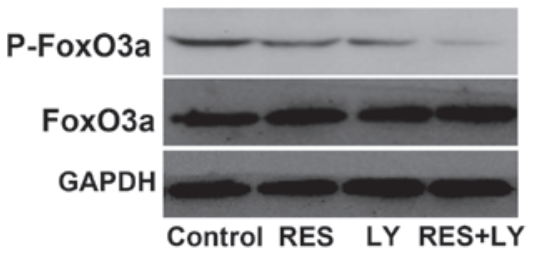

D

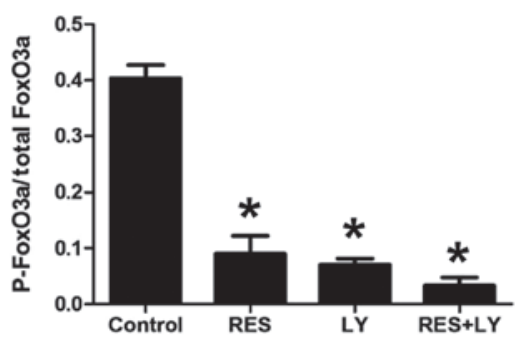

Figure 4. Effects of the inhibition of the PI3K pathway on the levels of phosphorylated and total Akt and FoxO3a in HepG2 cells. HepG2 cells were incubated in medium for $24 \mathrm{~h}$ (control group), resveratrol (100 $\mu \mathrm{M}$ ) for $48 \mathrm{~h}$ (RES group), $10 \mu \mathrm{mol} / 1 \mathrm{LY} 294002$ for $30 \mathrm{~min}$ prior to exposure to resveratrol (100 $\mu \mathrm{M})$ for $48 \mathrm{~h}$ (RES + LY group), or treated with $10 \mu \mathrm{mol} / 1 \mathrm{LY} 294002$ for $30 \mathrm{~min}$ followed by a 24-h culture (LY group). (A and B) Western blot analysis was used to detect changes in the phosphorylation of Akt and FoxO3a. (C and D) Relative levels of p-FoxO3a vs. total FoxO3a and p-Akt vs. total Akt in each sample as determined by densitometry. Values are expressed as the mean \pm standard error of the mean $(n=3)$. $P<0.05$, compared with the control group. RES, resveratrol; p-FoxO3a, phosphorylated forkhead box O3a; LY, PI3K inhibitor LY294002; PI3K, phosphoinositide-3 kinase.
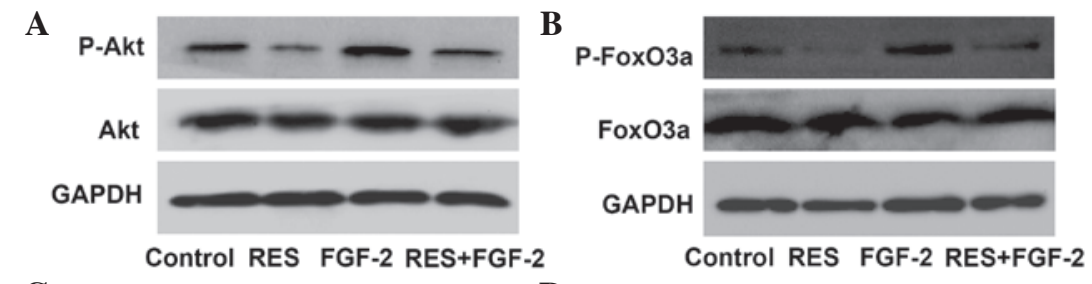

C

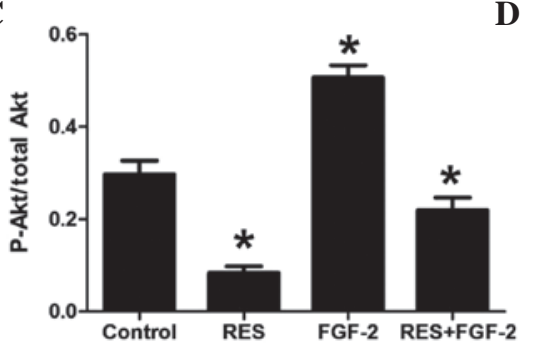

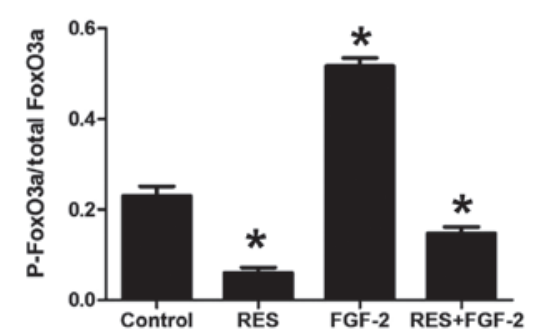

Figure 5. Resveratrol is effective in HepG2 cells stimulated with FGF-2. HepG2 cells were incubated in medium for $24 \mathrm{~h}$ (control group), resveratrol (100 $\mu \mathrm{M}$ ) for $48 \mathrm{~h}$ (RES group), $10 \mathrm{ng}$ FGF-2 for 30 min prior to exposure to resveratrol (100 $\mu \mathrm{M}$ ) for $48 \mathrm{~h}$ (RES + FGF-2 group), or treated with $10 \mathrm{ng}$ FGF-2 for 30 min followed by a 24-h culture (FGF-2). (A and B) Phosphorylation of Akt and FoxO3a was analyzed by immunoblotting. (C and D) Relative levels of p-FoxO3a vs. total FoxO3a and p-Akt vs. total Akt in each sample as determined by densitometry. Values are expressed as the mean \pm standard error of the mean ( $\mathrm{n}=3$ ). ${ }^{*} \mathrm{P}<0.05$, compared with the control group. FGF-2, fibroblast growth factor 2; RES, resveratrol; p-FoxO3a, phosphorylated forkhead box O3a.

PI3K/Akt/FoxO3a pathway in resveratrol-induced apoptosis, the phosphorylation levels of $\mathrm{Akt}$ and $\mathrm{FoxO} 3$ a were determined in HepG2 cells. Fig. 3 shows that resveratrol decreased the phosphorylation of Akt and FoxO3a in a time-dependent manner. The levels of phosphorylated Akt and $\mathrm{FoxO} 3 \mathrm{a}$ were significantly decreased at $24 \mathrm{~h}$, and were almost completely abolished following $48 \mathrm{~h}$ of resveratrol treatment.

EffectsofresveratroltreatmentonthePI3K/Akt/FoxO3apathway. To further confirm the involvement of the PI3K/Akt/FoxO3a pathway in resveratrol-induced apoptosis, the PI3K inhibitor LY294002 was used (Fig. 4). Treatment with resveratrol or LY294002 alone significantly decreased the phosphorylation of Akt and FoxO3a, which was further decreased by the combination of LY294002 and resveratrol.

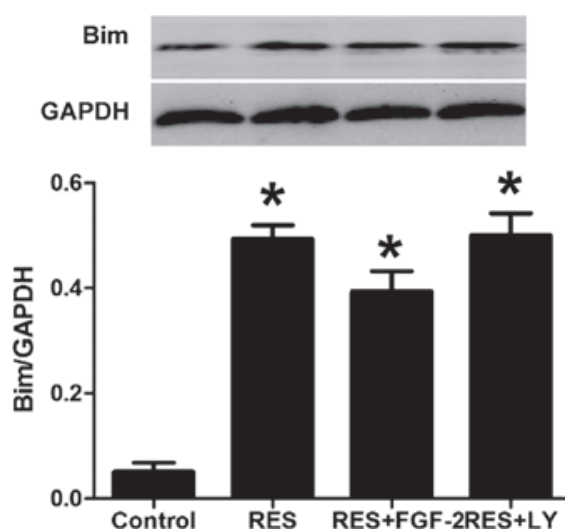

Figure 6. Effect of resveratrol on Bim expression. Values are expressed as the mean \pm standard error of the mean $(n=3)$. ${ }^{*} \mathrm{P}<0.05$, compared with the control group. FGF-2, fibroblast growth factor 2; RES, resveratrol; LY, phosphoinositide-3 kinase inhibitor LY294002. 
In addition, the effect of resveratrol on the PI3K/Akt/FoxO3a pathway was assessed under stimulation with FGF-2. As shown in Fig. 5, FGF-2 significantly increased the phosphorylation levels of Akt and FoxO3a. Furthermore, compared with FGF-2 treatment alone, combined treatment with FGF-2 and resveratrol significantly decreased the levels of phosphorylated Akt and FoxO3a.

Resveratrol treatment increases Bim expression in Hep G2 cells. Western blot analysis showed that resveratrol treatment significantly induced the expression of Bim protein. In addition, as shown in Fig. 6, resveratrol-induced Bim expression was further enhanced by co-treatment with FGF-2 or LY294002.

\section{Discussion}

Hepatocellular carcinoma is the most common type of liver cancer and to date, treatments have remained ineffective (2). Resveratrol is a nutrient compound with anti-cancer activity, and has been demonstrated to inhibit the initiation and development of cancer (6). The ability to cause cell-growth arrest is an important feature and a basic characteristic of cancer chemopreventive agents (19). Resveratrol is able to inhibit the proliferation of tumor cells via interfering with the progression of the cell cycle (9) and inhibiting the synthesis of DNA (20). However, the underlying molecular mechanism of resveratrol-induced apoptosis in hepatocellular carcinoma has remained to be fully elucidated.

FoxO3a is an important transcription factor and tumor suppressor; it is activated by stress signaling, which results in cell apoptosis $(12,13)$. Akt can promote FoxO3a phosphorylation, leading to FoxO3a translocation from the nucleus to the cytoplasm, which de-activates FoxO3a; conversely, inhibition of Akt promotes de-phosphorylation of FoxO3a, resulting in nuclear translocation of FoxO3a (13). The present study provided clear evidence of resveratrol-induced FoxO3a translocation to the nucleus. In addition, the present study showed that treatment of HepG2 cells with resveratrol significantly reduced the de-phosphorylated forms of Akt and FoxO3a. These results indicated that resveratrol inhibited the Akt survival pathway, leading to the promotion of FoxO3a translocation.

In order to further investigate the role of Akt in resveratrol-induced HepG2-cell apoptosis, HepG2 cells were treated with the PI3K/Akt inhibitor LY294002, or with FGF-2, which is able to activate Akt and promote cancer-cell viability. LY294002 significantly decreased the phosphorylation of Akt and FoxO3a, while FGF-2 significantly increased the phosphorylation of Akt and FoxO3a. In addition, the phosphorylation of Akt and FoxO3a was inhibited or enhanced to an even greater extent in the resveratrol + LY294002 and resveratrol + FGF-2 groups, respectively. This result further confirmed that resveratrol induced FoxO3a translocation via Akt signaling.

Tzivion et al (21) reported that FoxO3a was able to promote the expression of pro-apoptotic gene Bim. While the role of FoxO3a has been extensively studied in certain types of cancer, including thyroid cancer cell lines (22), little is known with regard to the effect of resveratrol on hepatocellular carcinoma and the possible implication of the FoxO3a. Roy et al (23) has reported that resveratrol inhibited the phosphorylation of FoxO3a and with simultaneous upregulation of the expression of Bim in in vitro cancer models. The present study was the first, to the best of our knowledge, to show that resveratrol-induced HepG2-cell apoptosis was mediated via the FoxO3a/Bim pathway.

In conclusion, the results of the present study indicated that resveratrol induces apoptosis in HepG2 liver cancer cells through modulation of the Akt/FoxO3a/Bim pathway. The study provided evidence that resveratrol is a promising drug against hepatocellular carcinoma.

\section{Acknowledgements}

This study was supported by a grants from the Graduate Student Research Innovation Project of Hunan province (no. CX2013B397).

\section{References}

1. Jemal A, Bray F, Center MM, Ferlay J, Ward E and Forman D: Global cancer statistics. CA Cancer J Clin 61: 69-90, 2011.

2. Gao Q, Shi Y, Wang X, Zhou J, Qiu S and Fan J: Translational medicine in hepatocellular carcinoma. Front Med 6: 122-133, 2012.

3. Pervaiz S and Holme AL: Resveratrol: Its biologic targets and functional activity. Antioxid Redox Signal 11: 2851-2897, 2009.

4. Foti Cuzzola V, Ciurleo R, Giacoppo S, Marino S and Bramanti P: Role of resveratrol and its analogues in the treatment of neurodegenerative diseases: Focus on recent discoveries. CNS Neurol Disord Drug Targets 10: 849-862, 2011.

5. Zordoky BN, Robertson IM and Dyck JR: Preclinical and clinical evidence for the role of resveratrol in the treatment of cardiovascular diseases. Biochim Biophys Acta 1852: 1155-1177, 2015.

6. Signorelli P and Ghidoni R: Resveratrol as an anticancer nutrient: Molecular basis, open questions and promises. J Nutr Biochem 16: 449-466, 2005.

7. Wood JG, Rogina B, Lavu S, Howitz K, Helfand SL, Tatar M and Sinclair D: Sirtuin activators mimic caloric restriction and delay ageing in metazoans. Nature 430: 686-689, 2004.

8. Delmas D, Jannin B, Cherkaoui Malki M and Latruffe N Inhibitory effect of resveratrol on the proliferation of human and rat hepatic derived cell lines. Oncol Rep 7: 847-852, 2000.

9. Parekh P, Motiwale L, Naik N and Rao KV: Downregulation of cyclin D1 is associated with decreased levels of p38 MAP kinases, Akt/PKB and Pak1 during chemopreventive effects of resveratrol in liver cancer cells. Exp Toxicol Pathol 63: 167-173, 2011.

10. Weng SC, Kashida Y, Kulp SK, Wang D, Brueggemeier RW, Shapiro CL and Chen CS: Sensitizing estrogen receptor-negative breast cancer cells to tamoxifen with OSU-03012, a novel celecoxib-derived phosphoinositide-dependent protein kinase-1/Akt signaling inhibitor. Mol Cancer Ther 7: 800-808, 2008.

11. Khan A, Aljarbou AN, Aldebasi YH, Faisal SM and Khan MA: Resveratrol suppresses the proliferation of breast cancer cells by inhibiting fatty acid synthase signaling pathway. Cancer Epidemiol 38: 765-772, 2014.

12. Nho RS and Hergert P: FoxO3a and disease progression. World $\mathrm{J}$ Biol Chem 5: 346-354, 2014.

13. Liu MH, Yuan C, He J, Tan TP, Wu SJ, Fu HY, Liu J, Yu S, Chen YD, Le QF, et al: Resveratrol Protects PC12 cells from high glucose-induced neurotoxicity via PI3K/Akt/FoxO3a pathway. Cell Mol Neurobiol 35: 513-522, 2015.

14. Polter A, Yang S, Zmijewska AA, van Groen T, Paik JH, Depinho RA, Peng SL, Jope RS and Li X: Forkhead box, class O transcription factors in brain: Regulation and behavioral manifestation. Biol Psychiatry 65: 150-159, 2009.

15. Su JL, Cheng X, Yamaguchi H, Chang YW, Hou CF, Lee DF, Ko HW, Hua KT, Wang YN, Hsiao M, et al: FOXO3a-dependent mechanism of E1A-Induced chemosensitization. Cancer Res 71: 6878-6887, 2011.

16. Wang JG, Zheng XX, Zeng GY, Zhou YJ and Yuan H: Purified vitexin compound 1 induces apoptosis through activation of FOXO3a in hepatocellular carcinoma. Oncol Rep 31: 488-496, 2014. 
17. Carbajo-Pescador S, Steinmetz C, Kashyap A, Lorenz S, Mauriz JL, Heise M, Galle PR, González-Gallego J and Strand S: Melatonin induces transcriptional regulation of Bim by FoxO3a in HepG2 cells. Br J Cancer 108: 442-449, 2013.

18. He L, Yang X, Cao X, Liu F, Quan M and Cao J: Casticin induces growth suppression and cell cycle arrest through activation of FOXO3a in hepatocellular carcinoma. Oncol Rep 29: 103-108, 2013.

19. Izzotti A: Molecular medicine and the development of cancer chemopreventive agents. Ann N Y Acad Sci 1259: 26-32, 2012.

20. Pirola L and Fröjdö S: Resveratrol: One molecule, many targets. IUBMB Life 60: 323-332, 2008.
21. Tzivion G, Dobson M and Ramakrishnan G: FoxO transcription factors; Regulation by AKT and 14-3-3 proteins. Biochim Biophys Acta 1813: 1938-1945, 2011.

22. Hong ZY, Lee HJ, Shin DY, Kim SK, Seo M and Lee EJ: Inhibition of Akt/FOXO3a signaling by constitutively active FOXO3a suppresses growth of follicular thyroid cancer cell lines. Cancer Lett 314: 34-40, 2012.

23. Roy SK, Chen Q, Fu J, Shankar S and Srivastava RK Resveratrol inhibits growth of orthotopic pancreatic tumors through activation of FOXO transcription factors. PLoS One 6: e25166, 2011. 